The development of a triple-channel separator for particle removal with self-pumping oscillating flow

This article has been downloaded from IOPscience. Please scroll down to see the full text article.

2007 J. Micromech. Microeng. 17439

(http://iopscience.iop.org/0960-1317/17/3/004)

The Table of Contents and more related content is available

Download details:

IP Address: 140.112.113.225

The article was downloaded on 19/12/2008 at 07:44

Please note that terms and conditions apply. 


\title{
The development of a triple-channel separator for particle removal with self-pumping oscillating flow
}

\author{
C J Lee, H J Sheen, H C Chu, C J Hsu and T H Wu \\ Institute of Applied Mechanics, National Taiwan University, Taipei 106, Taiwan, \\ Republic of China \\ E-mail: sheenh@ntu.edu.tw
}

Received 9 November 2006, in final form 5 January 2007

Published 30 January 2007

Online at stacks.iop.org/JMM/17/439

\begin{abstract}
The results of a new triple-channel separator with dual functions of fluid pumping and particle removal are presented in this paper. The separator was made through a MEMS fabrication process using only one photo mask. A valve-less micropump was connected to a triple-channel at a downstream position, and the oscillating flow in the micro-channel was produced by the micropump. An important characteristic of the separator is its oscillating flow, which produces two vortices at the trifurcate zone. These vortices served as obstacles to increase the flow resistance of the center channel. Based on the rotating direction of these vortices, the particles were driven towards side channels to achieve the removal effect. Micro-particle image velocimetry ( $\mu$-PIV) with an external trigger was used to measure the flow characteristics of the vortices. Streamtrace patterns were obtained at the trifurcate zone in a time period. Image processing was used to count the number of particles and to analyze the removal efficiency. The optimal removal efficiency (close to $100 \%$ ) is obtained at a driving frequency of $1.5 \mathrm{kHz}$ and a divaricated angle of $25^{\circ}$. This study indicates that the positions of the vortices provided improved removal performance with respect to the driving frequency of the PZT (piezoelectric zirconium titanate) plate and the divaricated angle of the triple-channel.
\end{abstract}

\section{Introduction}

Particle separators are essential components in micro-total analysis systems ( $\mu$-TAS) [1-4] and are widely used in biochemical [5, 6] and bio-medical [7-10] applications. One of the key issues in developing these systems is how simple and high separation performance can be achieved. In previous studies, two noncontact technologies, dielectrophoresis (DEP) and acoustic wave, were the most frequently used approaches for particle separation in suspension. DEP is the lateral movement of particles induced by polarization effects in nonuniform electric fields [11]. In the DEP devices, mixing particles are injected into a separation chamber and an electric field is applied for separation [12-18]. The particles are separated on the basis of sizes and DEP properties. Theoretical studies on acoustic radiation forces $[19,20]$ indicated that a rigid and compressible sphere in a nonviscous fluid can be trapped in such a field. The efficacy of using acoustic force to concentrate small particles in suspension has also been evaluated theoretically and experimentally [21-24]. That this method could be used to separate particles continuously in a micro-channel was proposed in [25-27]. Those devices adopted acoustic wave and operated in a half wavelength standing wave field.

In addition, a special design in the geometry of the microchannel, such as a pinched flow fractionation (PFF) [28, 29], has been used to separate and filter particles of different sizes. Filtration has a critical drawback, namely clogging in the channel, which makes it impossible to separate repetitively. A new hydrodynamic filtration method [30] was developed to avoid this clogging problem, but precise channel geometry and flow rate controls are necessary. While a variety of separators have been studied with DEP, acoustic wave and PFF in the laminar flow, the oscillating flow generated from a 

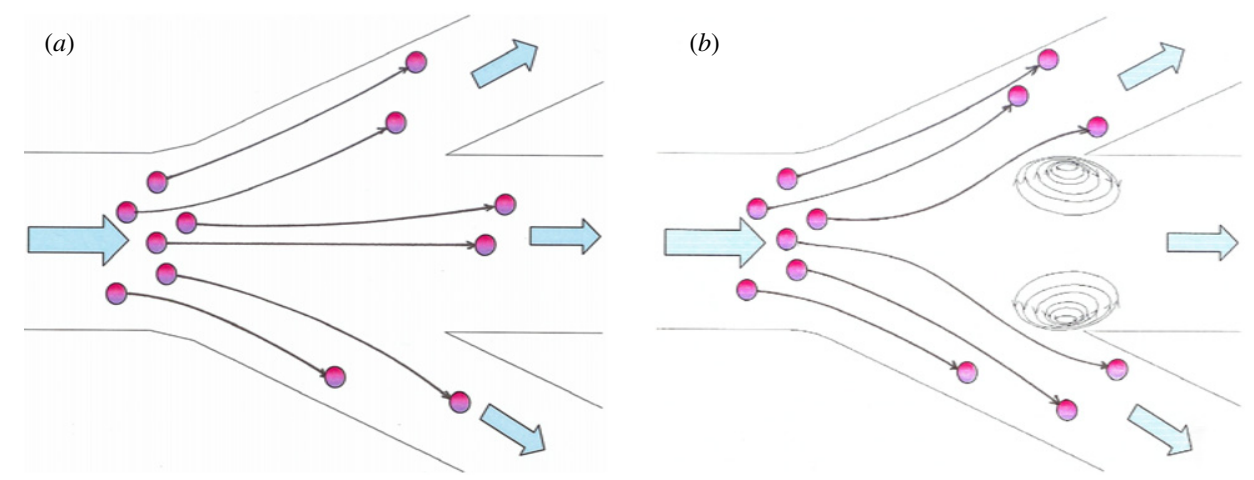

Figure 1. Principle of particle removal by vortices. Schematic diagrams showing particle behavior at the trifurcate zone: $(a)$ the particles go to the center and side channels uniformly in a steady flow. (b) Two vortices are generated upstream from the center channel in an oscillating flow. The vortices increase the flow resistance of the center channel, and the rotating directions of the vortices lead the particles to move towards the side channels.

micropump has received much less attention. However, in the miniaturization of bio-detecting applications, the micropump is a necessary component in the system. The flow pattern in the micro-channel is an oscillating flow, not a laminar flow. The flow characteristics of an oscillating flow in the micro-channel were used to achieve particle removal without an external pumping source. The distinct feature makes the present device ideal for a portable $\mu$-TAS or lab-on-a-chip.

In this paper, we propose a new triple-channel separator for particle removal with a self-pumping function to remove the particles from a fluid suspension. In the present study, we first estimated the efficacy of pumping performance and then compared removal efficiency with the driving voltage and frequency of the PZT plate and the initial concentration of the suspension. The flow visualization technique was used to measure the flow characteristics of an oscillating flow to observe the effects of the vortices in this device.

\section{Principle of operation}

This study integrates a PZT valve-less micropump and a trifurcate zone into a new triple-channel separator for particle removal. The suspension can be pumped from the inlet and the particles removed into the side channels. The net flow rate of the micropump was obtained under a time period of an oscillating PZT plate. The detailed operating principles of the valve-less micropump were presented in [31]. The diffuser/nozzle elements were placed on each side of the oscillating chamber with a vibrating membrane. The membrane produces a change of the volume of the chamber which is connected with the diffuser/nozzle, and a net flow towards downstream direction is obtained. The use of MEMS processes, including HNA (hydrofluoric, nitric and acetic acids), DRIE (deep reactive ion etching), ICP (inductively coupled plasma) etching and thermoplastic replication, were proposed for use in the fabrication of valve-less micropumps [32-35].

The oscillating flow, which is generated by the micropump, is used to induce vortices upstream from the inlet of the center channel. These vortices can be utilized for the removal of the dispersed particles from the suspension.
From the results of flow visualization, the following two flow characteristics of the oscillating flow were observed. Firstly, the maximum velocity of the flow occurred near the wall of the micro-channel. The velocity distribution is similar to the velocity profile in a circular pipe that was presented in [36]. The velocity profile was very different from the Poiseuille flow, which is in a parabolic distribution and in which the maximum velocity occurs in the center of the channel. Secondly, the vortices were generated in the neighborhood of the channel wall when the oscillating flow passed through the diffuser or an obstacle. There are two operation modes, pump mode and supply mode, in one operating cycle of the present device. For the pump mode, the oscillating chamber is decreased in volume and the fluid flows through the main channel into the trifurcate zone. However, the reverse flow is obtained in the supply mode when the volume of the oscillating chamber is increased. The vortices occurred as the supply mode and the pump mode periodically alternated during a time period of the oscillating flow [37].

For a steady flow, as the fluids flow through the trifurcate zone as shown in figure $1(a)$, the particles move to the center and the side channels uniformly. However, in the oscillating flow, two vortices are generated upstream from the center channel, as shown in figure 1(b). The vortices increase the flow resistance of the center channel, and the rotating directions of the vortices lead the particles to move towards the side channels. By this phenomenon, a large portion of the particles can be removed from the main channel after passing through the trifurcated zone. Therefore, the positions of the vortices have a significant effect on the removal efficiency, and such a position is influenced with the divaricated angle of the triplechannel. The removal efficiency was estimated with two divaricated angles in this study, and various driving voltages and frequencies of the PZT plate were also investigated to optimize this separator.

\section{Design and fabrication}

The present device was designed based on the efficiency of the pump and particle removal. The main factor of the pumping efficiency is the geometry of the diffuser of the valve-less 

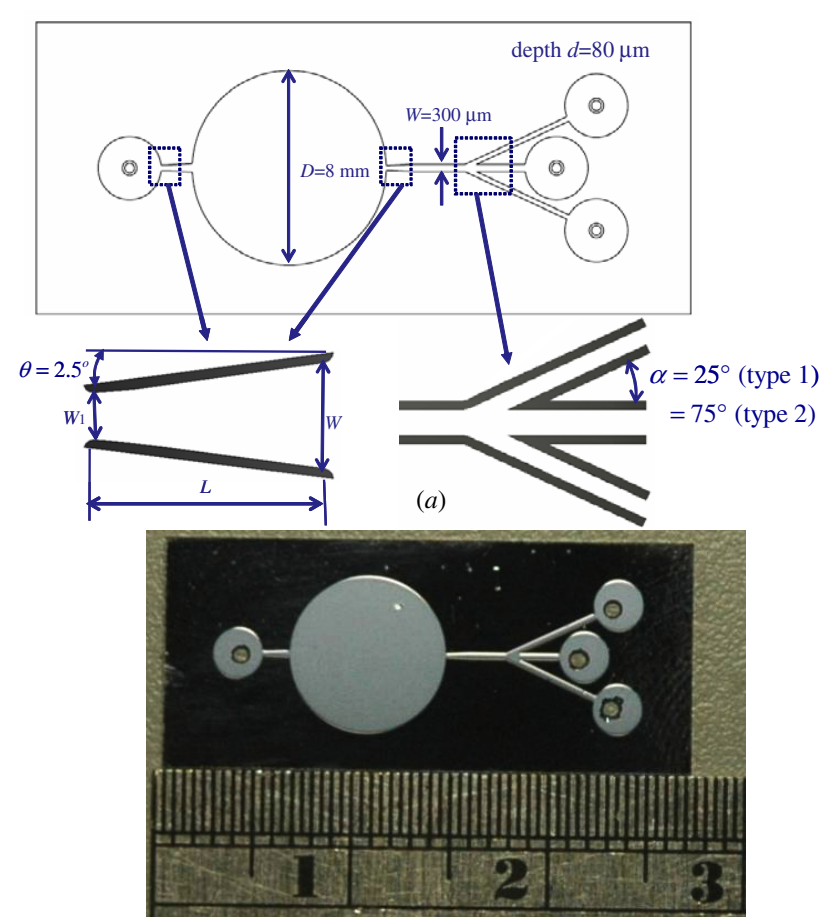

(b)

Figure 2. (a) Schematic illustration of the triple-channel separator for particle removal, where the length of the diffuser is $L$, the width of the micro-channel is $W$, the neck width is $W_{1}$, the divergence angle is $\alpha$ and the diameter of the vibrating chamber is $D$. (b) A photograph of the present separator shows one inlet, the oscillating chamber, the trifurcate zone and three outlets.

micropump. A schematic illustration of the triple-channel separator is shown in figure $2(a)$. The three key geometrical parameters of a diffuser are the inlet width $W_{1}$, diffuser angle $2 \theta$ and diffuser length $L$. In this experiment, the inlet width was $200 \mu \mathrm{m}$, the diffuser angle was $5^{\circ}$, the diffuser length was $1285 \mu \mathrm{m}$, the width of the micro-channel, $W$, was $300 \mu \mathrm{m}$, the depth of the micro-channel, $d$, was fixed to $80 \mu \mathrm{m}$ and the diameter of the oscillating chamber, $D$, was $8 \mathrm{~mm}$. The slenderness, $L / W_{1}$, was designed to be 6.4. A photograph of this device with one inlet and three outlets is shown in figure $2(b)$. The overall size of this separator is approximately $10 \times 25 \times 1.0 \mathrm{~mm}$. A PZT plate, with a thickness of $200 \mu \mathrm{m}$, was placed onto a chamber with epoxy to constitute the whole device. The divaricated angle, $\alpha$, of the triple-channel was designed to be $\alpha=25^{\circ}$ for type I and $\alpha=70^{\circ}$ for type II. Different angles were used to estimate the influence on the positions of two vortices.

The fabrication process of this study was simple since only one photo mask, one ICP etching step and anodic glass bonding were required. The fabrication process of the separator is shown in figure 3. This device was fabricated in a $500 \mu \mathrm{m}$ thick silicon wafer using ICP etching. The main benefit of the ICP etching technique is that it precisely controls the pattern and dimensions of the diffuser and triple-channel. Anodic bonding with a sodium glass wafer (Corning 7740, thickness of $500 \mu \mathrm{m}$ ) was carried out at a temperature of $400{ }^{\circ} \mathrm{C}$ when an external voltage in the range of 400-800 V was applied.

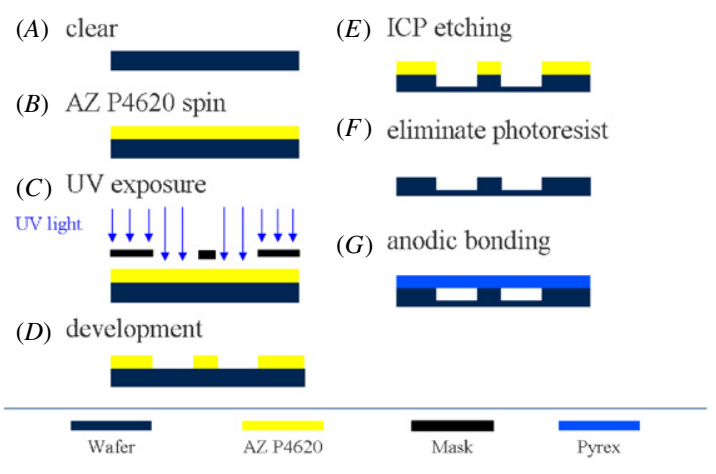

Figure 3. Schematic illustration of the simplified fabrication process for the triple-channel separator.

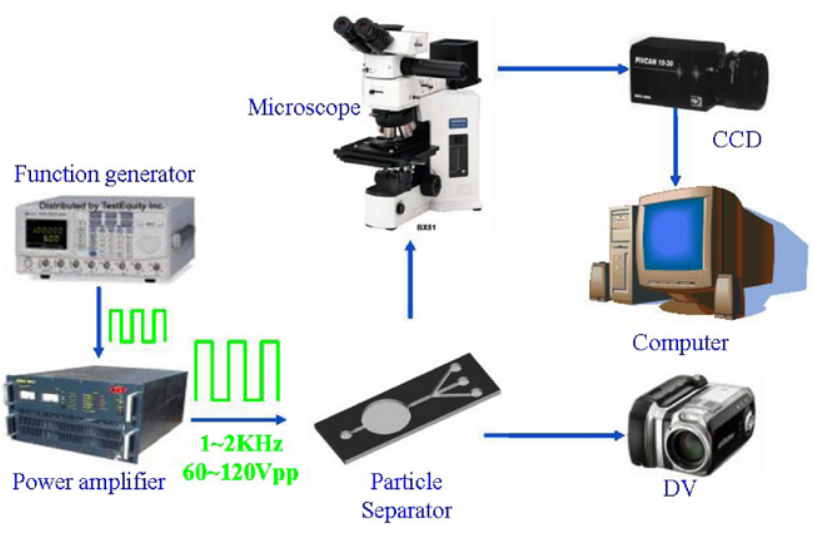

Figure 4. Schematic illustration of the experimental system.

\section{Experimental apparatus and measurements}

Figure 4 presents a schematic of the experimental system used for the operation of the triple-channel separator. A function generator generated a square wave, from 900 to $1400 \mathrm{~Hz}$, to a power amplifier, and the amplifier output a voltage, from 60 to $120 V_{\mathrm{PP}}$, to drive the PZT plate in harmonic oscillation. The output voltage and frequency from the power amplifier were monitored by an oscilloscope. While the separator was turned on, the fluid levels changed due to the back pressure, and the pressure differences between fluid levels of the inlet and outlets were recorded with a digital camera. An image analysis was made to evaluate the flow rate. During the particle removal tests, the separator was placed under an optical microscope, and the motion of the particles in the trifurcate zone was observed and recorded using a high-sensitivity CCD. Finally, image processing was applied to count the number of particles to obtain the removal efficiency. Noise in these images was the main error factor during the counting process and a binary process was performed to increase the signal-to-noise ratio (SNR) in this study. Furthermore, the particles gathered in the suspension were another error factor. A red fluorescent polymer microsphere (Duke, 36-2B) that can be dispersed in aqueous media was used in the experiment. The particles have a density of $1.05 \mathrm{~g} \mathrm{~cm}^{-3}$ and a diameter of $6 \mu \mathrm{m}$ with standard deviation less than $16 \%$.

In addition, the $\mu$-PIV technology with an external trigger was applied to investigate the flow characteristics of the 


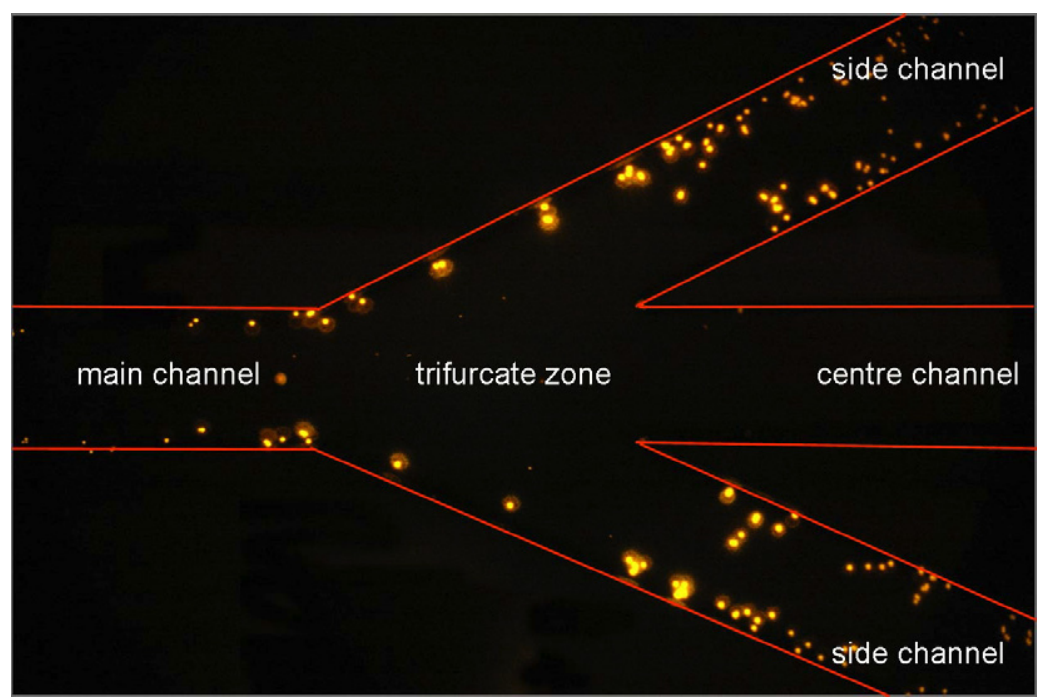

Figure 5. Top view microscope photograph of particle removal at the trifurcate zone. The driving voltage of the PZT plate is $80 V_{\mathrm{PP}}$ and the frequency is $1.3 \mathrm{kHz}$. (red lines are the walls of the micro-channels).

vortices at the trifurcate zone. For flow visualization, another fluorescent particle (with a diameter of $1 \mu \mathrm{m}$, Duke R0-100) was applied to obtain high resolution images of the flow pattern. This particle was excited by an Nd-YAG doublepulsed laser with a wavelength of $532 \mathrm{~nm}$. A $10 \times$ object lens was used to collect the fluorescence emissions. The illuminated particle images were captured by a high-sensitivity $\mathrm{CCD}$, and software with a cross-correlation function was used to observe the displacement of the same particle between two images in a short time. The velocity of the moving particles could be computed by the time difference between the two laser triggers, and thus the flow characteristics of the vortices in the trifurcate zone could be obtained. A detailed description of the operating principle of the $\mu$-PIV has been presented in [37-40].

\section{Results and discussion}

As can be seen in figure 5, the suspension was pumped from the main channel to the trifurcate zone, and the particles were removed into side channels with a driving voltage of $80 V_{\mathrm{PP}}$, a driving frequency of $1.3 \mathrm{kHz}$ and a divaricated angle of $25^{\circ}$. Most of the particles were moving towards the side channels instead of into the center channel. The removal efficiency approached $100 \%$. In this study, the performances of the pumping fluid and the particle removal in this triple-channel separator were estimated, and then the flow characteristics of the vortices were investigated by flow visualization.

\subsection{Pumping efficiency}

As for the pump efficiency, the geometry of the channel, the driving voltage and the frequency affect the flow rate provided by the separator. In this experiment, the inlet and outlets had the same fluid levels initially. During operation, these levels changed due to the pump pressure, and the differences between fluid levels of the inlet and outlets were measured.

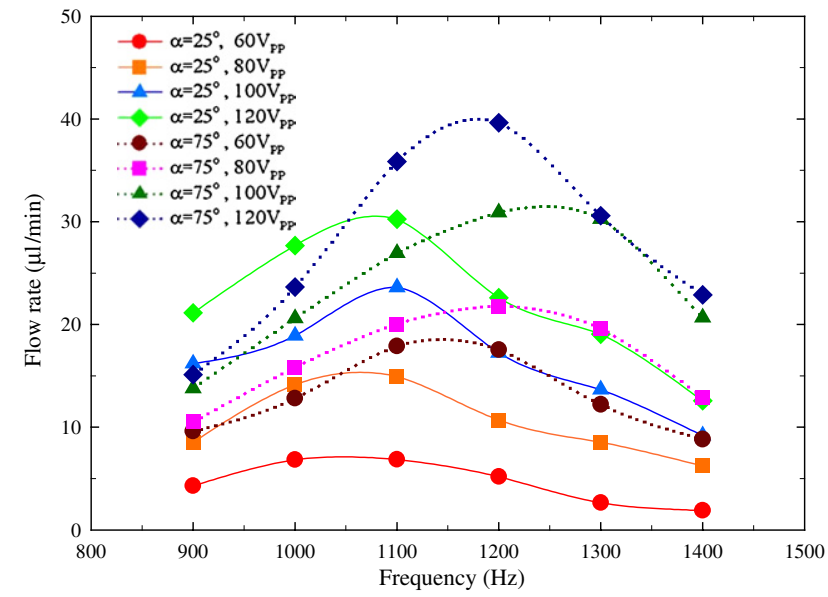

Figure 6. Flow rates of the two types of separators with various driving voltages and frequencies.

The flow rate, $\dot{Q}$, was calculated as

$$
\dot{Q}=\frac{\pi}{4} \frac{R^{2} \mathrm{~d} h}{t}
$$

where $R=1.125 \mathrm{~mm}$ is the inner radius of the glass tube. The duration time, $t$, was measured when an increment of height difference $\mathrm{d} h$ reached $3 \mathrm{~mm}$ from initial pumping. The flow rates were obtained by maintaining the same height to measure the duration at different driving voltages and frequencies. The effect of the two types of separators on flow rate with various driving voltages and frequencies was explored, and the results are shown in figure 6 . In general, the flow rate increases significantly as driving voltage is increased. From the testing process, the optimum operational frequencies for types I and II were found to be 1.1 and $1.2 \mathrm{kHz}$, respectively. When the driving voltage was $120 V_{\mathrm{PP}}$, the maximum flow rates attained were 30.2 and $39.6 \mu \mathrm{min}^{-1}$, respectively, for types I and II. The pump efficiency of type II was approximately $30 \%$ better than that of type I (at a constant driving voltage). 


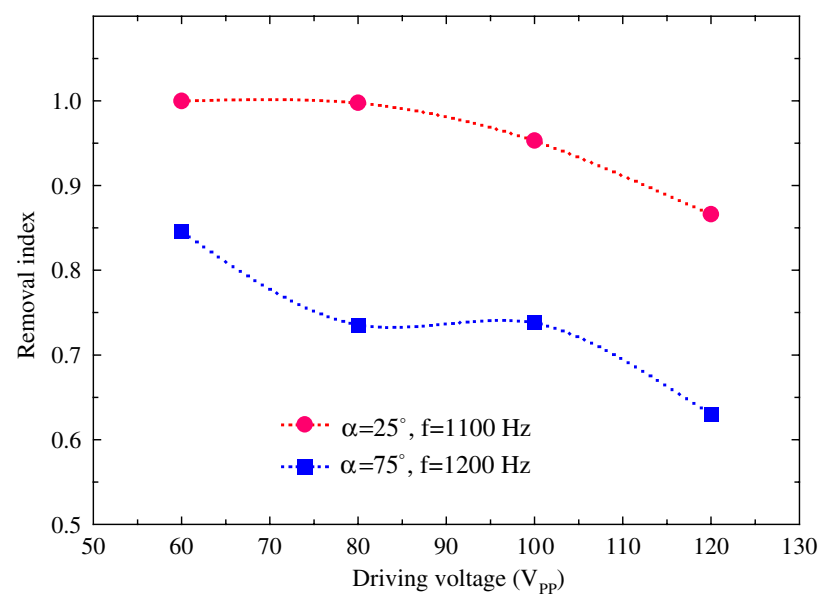

Figure 7. The removal indices of the two types of separators with various driving voltages.

(This figure is in colour only in the electronic version)

In order to investigate whether the flow pattern is laminar or turbulent, it is common to estimate the Reynolds number, $R e$, and compare it with the transition Reynolds number. The definition of the transition Reynolds number depends on the ratio between the length, $L$, and the hydraulic diameter, $D_{h}$ [41]. With small values of $L / D_{h}$, the transition Reynolds number, $R e_{t}$, is defined as

$$
R e_{t} \approx 30 \frac{L}{D_{h}}, \quad 1<L / D_{h}<70 .
$$

For this device, the ratio $L / D_{h}=10.17$, and the $R e_{t}$ is 305 . In this experiment, the values of $R e$ were $0.23-3.45$ at the average flow rate, and the value of $R e$ was 125 at the maximum velocity during the operating cycle. The fact that the values of $R e$ were much smaller than $R e_{t}$ clearly shows that the flow field was predominantly laminar.

\subsection{Removal efficiency}

The removal efficiency was determined as the ratio of the particles in the side channels to the total particles in the fluid injected from the main channel. To evaluate the removal efficiency, a removal index $R_{I}$ was defined in this study as

$$
R_{I}=1-\frac{A}{B}
$$

where $A$ is the particle number of the fluid collected from the center channel and $B$ is the particle number of the fluid injected from the main channel.

The relations between the driving voltage and the removal index under the optimal operating frequency at different separators are shown in figure 7 . The optimal removal efficiency for type $\mathrm{I}$ is $R_{I}=0.99$, and a great majority of particles move forward to the side channels; thus, a fluid with almost no particles can be obtained in the center channel. The optimal removal index is only $R_{I}=0.85$ for the type II separator. From the results, the removal efficiencies decrease as the driving voltages increase in the same separator. At higher driving voltages, the flow velocity is increased and the particles are provided with high kinetic energy to pass through the vortices. As a result, the blocking effect of the vortices is

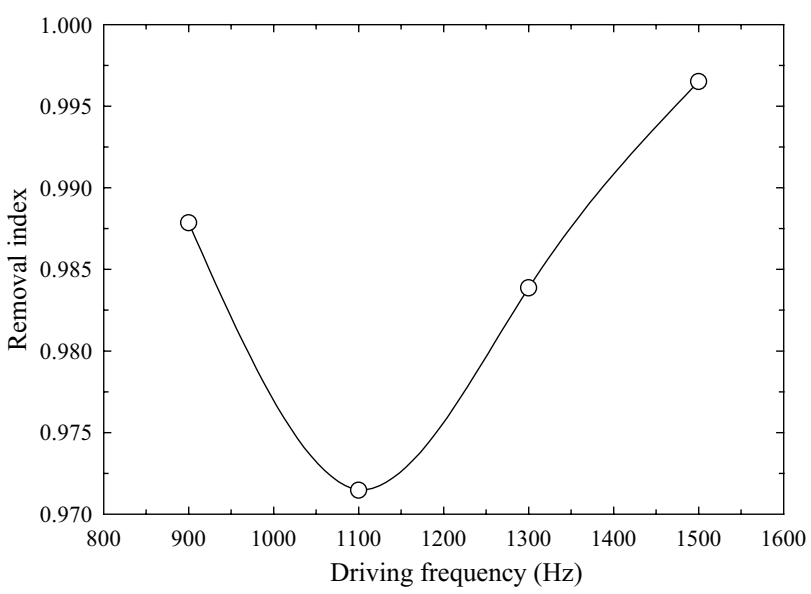

Figure 8. The removal indices of the type I separator with various driving frequencies.



Figure 9. The removal indices of the type I separator with various initial concentrations.

decreased, thus causing a drop in the removal efficiency. From figures 6 and 7, it can be seen that the removal efficiencies of type I are always better than those of type II. This means that the divaricated angle of the triple-channel has a decisive influence on the removal efficiency.

Figure 8 demonstrates the relation between the driving frequency and the removal index for type I when the driving voltage was kept at $80 V_{\mathrm{PP}}$. The removal index was minimized when the frequency was $1.1 \mathrm{kHz}$. This frequency is the same as the optimal operating frequency for type I. This result indicates that the lower removal efficiencies were induced by higher flow rates, since more fluid volume was squeezed by the deformed PZT plate. In figure 9, the removal index as a function of the initial concentration of the fluid is shown for the type I separator. The optimal frequency of $1.1 \mathrm{kHz}$ and a driving voltage of $80 V_{\mathrm{pp}}$ were used. A decrease of the removal efficiency with increasing initial concentration was observed. The distances between particles were reduced with higher concentrations, and the collisions of particles increased when the particles passed through the vortices, causing the particles to be moved to the center channel and thus reducing the removal efficiency. 
(a) $t=1 / 16 T$

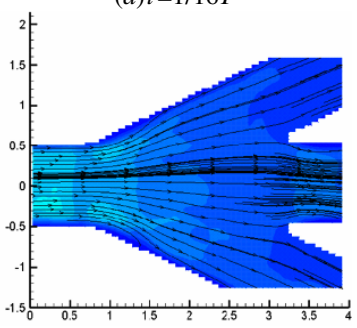

(e) $t=5 / 16 T$

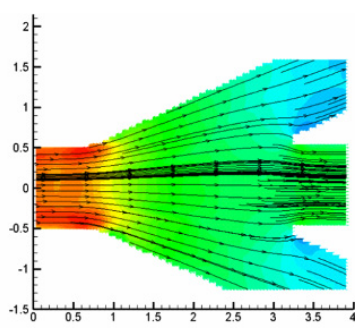

(i) $t=9 / 16 T$

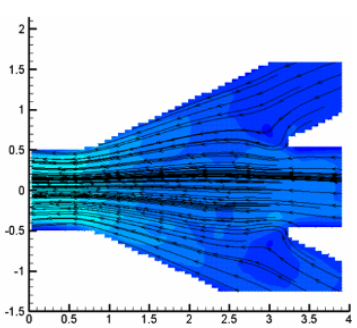

(m) $t=13 / 16 T$

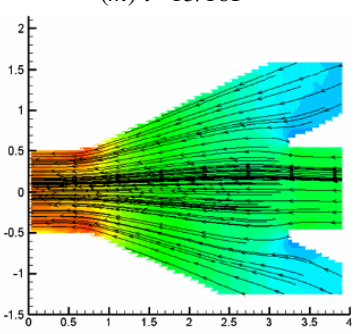

(b) $t=1 / 8 T$

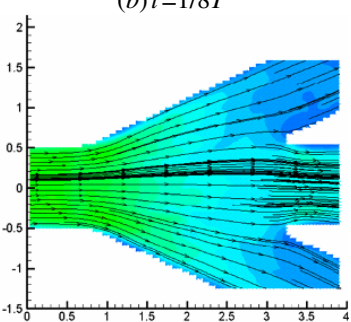

(f) $t=3 / 8 T$

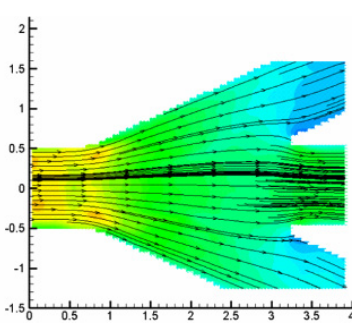

(j) $t=5 / 8 T$

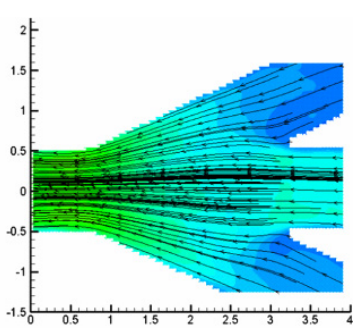

(n) $t=7 / 8 T$

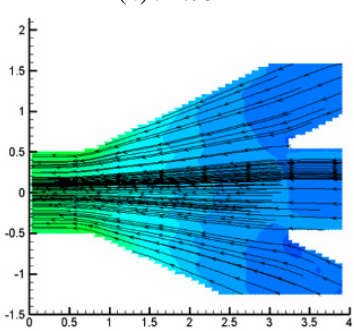

(c) $t=3 / 16 T$

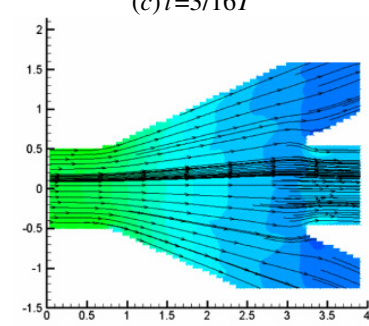

(g) $t=7 / 16 T$

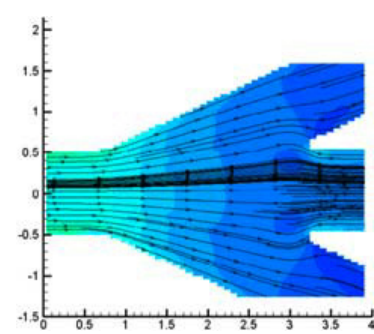

(k) $t=11 / 16 T$

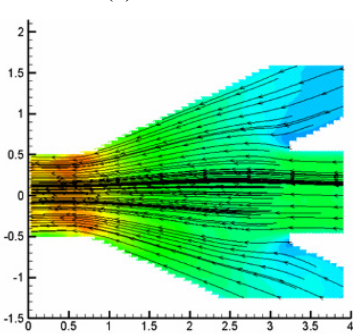

(o) $t=15 / 16 T$

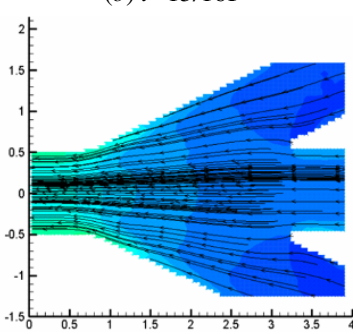

(d) $t=1 / 4 T$

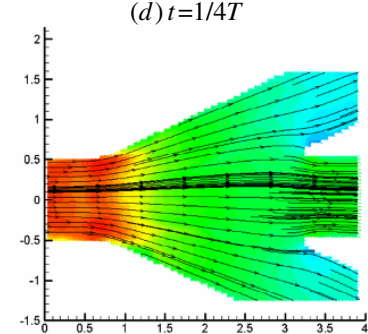

(h) $t=1 / 2 T$

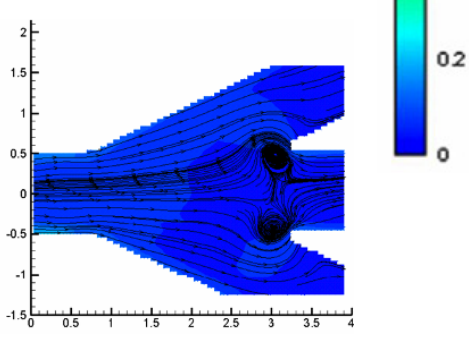

(l) $t=3 / 4 T$

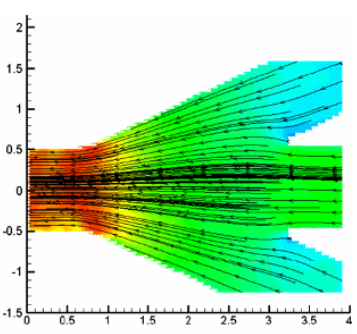

(p) $t=T$

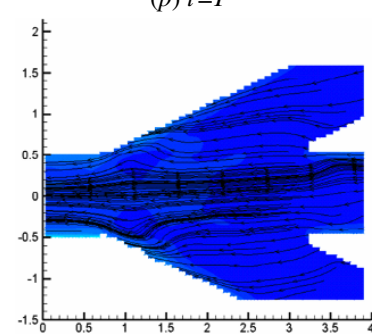

Figure 10. The streamtrace patterns in the type I separator in a time period. A velocity close to zero was observed as the pump mode switched into the supply mode and the vortices were produced in the trifurcate zone.

\subsection{Quantitative flow visualization}

The oscillating flow field in the trifurcate zone was successfully obtained by the externally triggered $\mu$-PIV. In the experiment, the velocity profiles depended on the oscillating frequency of the imposed pressure gradient and the dimension of the triplechannel. The images of flow visualization in figure 10 show the streamtrace patterns of the type I separator in a cycle at an interval of $1 / 16$ time period. The streamtrace patterns in the pump mode of an operating cycle are shown in figures 10(a)(h), and those in the supply mode are shown in figures 10(i)$(p)$. As shown in figures $10(h)$ and $(p)$, a velocity close to zero was observed as the pump mode and supply mode switched and the vortices were produced in the trifurcate zone.

The divaricated angle of the triple-channel had significant effects on the positions and the times of occurrence of the vortices. Figures 11 $(a)-(d)$ demonstrate the phenomena of the oscillating flows at $\alpha=25^{\circ}$ and $75^{\circ}$ in the trifurcate zone.
When the divaricated angle was $25^{\circ}$, the position of the vortices was upstream of the center channel, and the vortices occurred only while the pump mode was being switched into the supply mode as shown in figures $11(a)$ and $(b)$. The vortices worked as obstacles to reduce the inlet area and increased the flow resistance of the center channel. Furthermore, the rotating direction of the vortices was outward from the center channel, driving the particles towards the side channels to achieve the effect of removing the particles from the suspension.

However, when the divaricated angle was changed to $75^{\circ}$, the streamline patterns were chaotic in the trifurcate zone, as shown in figures $11(c)$ and $(d)$. The vortices occurred with alternation of the supply mode and pump mode. Furthermore, the positions of the vortices all occurred in the inlets of the side channels and occupied half the area of each channel. When the fluid flowed through the trifurcate zone, collisions among particles were induced by the vortices, and the particles were driven into all the outlets. Therefore, better removal efficiency 

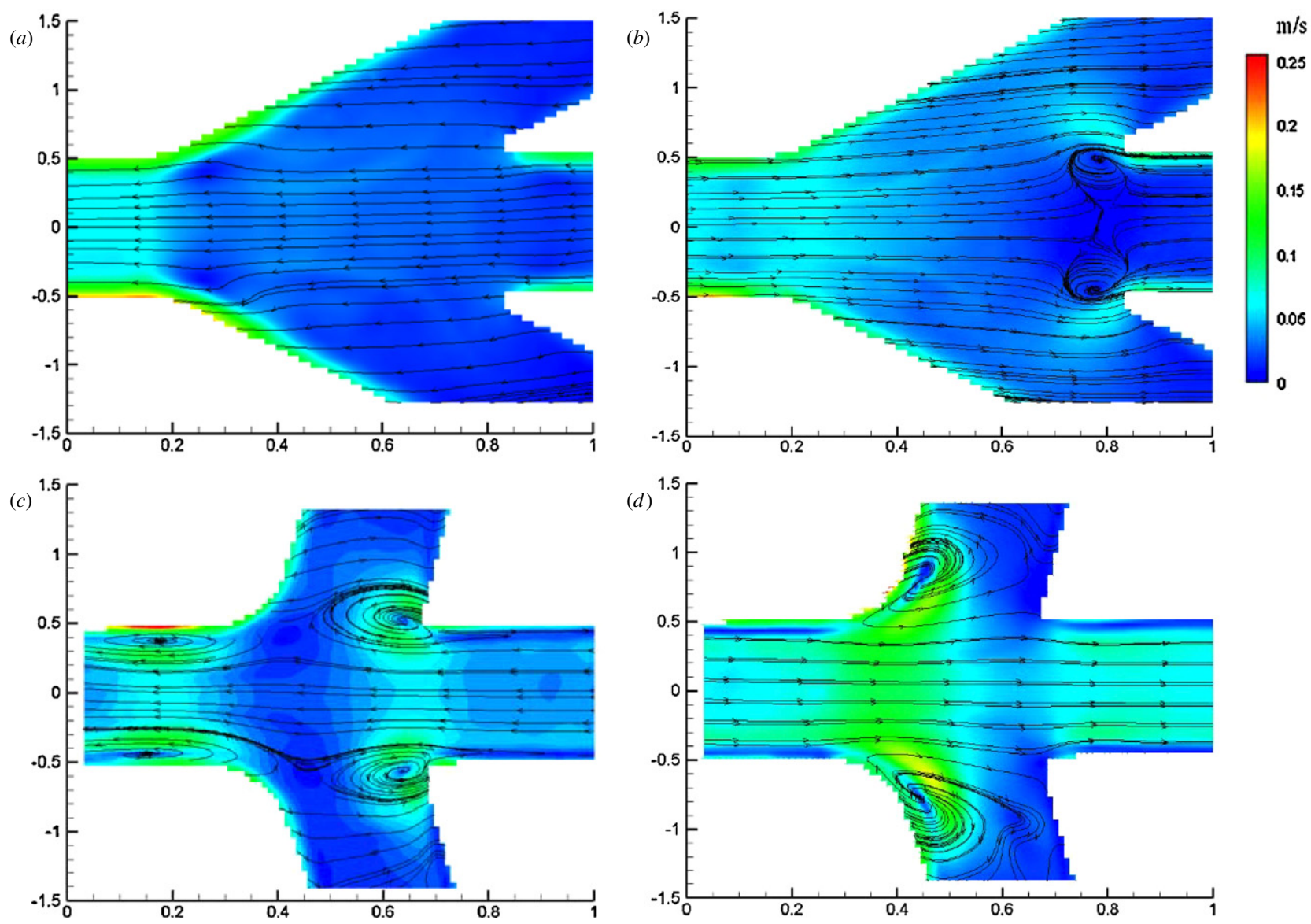

Figure 11. The streamtrace patterns of (a) supply to pump (b) pump to supply mode in type I and $(c)$ supply to pump $(d)$ pump to supply mode in type II.

was achieved with the smaller divaricated angle of the triplechannel in this study.

\section{Conclusions}

The objective of this study is to develop a triple-channel separator that can remove particles from a suspension. The device can be utilized for applications in bio-medical and chemical analyses, such as removing red blood cells from whole blood. This triple-channel separator for particle removal has been successfully demonstrated to be able to pump fluid and remove particles without any external pumping devices. This is a definite advantage over other separation techniques such as DEP, acoustic force and PFF, which require a syringe pump. The present device can be fabricated by a simple MEMS process which requires only one photo mask and one ICP etching process. This process not only simplifies the complicated processes of producing separators used in previous studies but also reduces the cost and enhances the yield. In this device, the particles can be removed from the suspension within $2 \mathrm{~mm}$, and the optimal removal efficiency is close to $100 \%$. The removal efficiencies were investigated with various driving voltages, frequencies and initial concentrations. Furthermore, the positions of occurrence of the vortices at the trifurcate zone with different divaricated angles were also observed in order to study the removal efficiency. Due to the reduced volume of the removal system, the present separator has the potential to be integrated with other detectors for uses in a miniaturized $\mu$-TAS in the future.

\section{Acknowledgments}

The authors gratefully acknowledge the financial support provided to this study by the National Science Council of Taiwan (NSC-95-2218-E-002-051-MY3) and the Ministry of Economic Affairs (94-EC-71-A-05-S1-0017), Taiwan, ROC.

\section{References}

[1] Stone H A, Stroock A D and Ajdari A 2004 Engineering flows in small devices: microfluidics toward a lab-on-a-chip Annu. Rev. Fluid Mech. 36 381-411

[2] Huang Y, Ewalt K L, Tirado M, Haigis R, Forster A, Ackley D, Heller M J, O'Connell J P and Krihak M 2001 Electric manipulation of bioparticles and macromolecules on microfabricated electrodes Anal. Chem. 73 1549-59

[3] Vilkner T, Janasek D and Manz A 2004 Micro total analysis systems. Recent developments Anal. Chem. 76 3373-85

[4] El-Ali J, Sorger P K and Jensen K F 2006 Cells on chips Nature 442 403-11

[5] Yasuda K, Haupt S S, Umemura S I, Yagi T, Nishida M and Shibata Y 1997 Using acoustic radiation force as a concentration method for erythrocytes J. Acoust. Soc. Am. $102642-5$ 
[6] Li, Paul C H and Harrison D J 1997 Transoprt, manipulation, and reaction of biological cells on-chip using electrokinetic effects Anal. Chem. 69 1564-8

[7] Huang L R, Cox E C, Austin R H and Sturm J C 2004 Continuous particle separation through deterministic lateral displacement Science 304 987-90

[8] Tegenfeldt J O, Prinz C, Cao H, Huang R L, Austin R H, Chou S Y, Cox E C and Sturm J C 2004 Micro- and nanofluidics for DNA analysis Anal. Bioanal. Chem. 378 1678-92

[9] Woolley A T and Mathies R A 1995 Ultrahigh-speed DNA sequencing using capillary array electrophoresis chips Proc. SPIE-Int. Soc. Opt. Eng. 2386 36-44

[10] Pal R et al 2005 An integrated microfluidic device for influenza and other genetic analyses Lab Chip 5 1024-32

[11] Pohl H A 1978 Dielectrophoresis: the behavior of neutral matter in nonuniform electric fields (Cambridge: Cambridge University Press)

[12] Huang Y, Joo S, Duhon M, Heller M, Wallace B and Xu X 2002 Dielectrophoretic cell separation and gene expression profiling on microelectronic chip arrays Anal. Chem. $743362-71$

[13] Doh I and Cho Y-H 2005 A continuous cell separation chip using hydrodynamic dielectrophoresis (DEP) process Sensors Actuators A 121 59-65

[14] Markx G H and Pethig R 1995 Dielectrophoretic separation of cells: continuous separation Biotechnol. Bioeng. 45 337-43

[15] Kentsch J, Durr M, Schnelle T, Gradl G, Muller T, Jager M, Normann A and Stelzle M 2003 Microdevices for separation, accumulation, and analysis of biological microand nanoparticles IEE Proc. Nanobiotechnol. 150 82-9

[16] Li Y and Kaler K V I S 2004 Dielectrophoretic fluidic cell fractionation system Anal. Chim. Acta 507 151-61

[17] Chou C F, Tegenfeldt J O, Bakajin O, Chan S S and Cox E C 2002 Electrodeless Dielectrophoresis of single- and double-stranded DNA Biophys. J. 83 2170-9

[18] Cummings E B and Singh A K 2000 Dielectrophoretic trapping without embedded electrodes, microfluidic devices and systems: III Proc. SPIE 4177 164-73

[19] King L V 1934 On the acoustic radiation pressure on spheres Proc. R. Soc. A 147 212-40

[20] Grokov L P 1962 On the force acting on a small particle in an aocustic field in an ideal fluid Sov. Phys.-Dokl. 6 773-5

[21] Tolt T L and Feke D L 1993 Separation of dispersed phases from liquids in acoustically driven chambers Chem. Eng. Sci. $48527-40$

[22] Mandralis Z, Bolek W, Burger W, Benes E and Feke D L 1994 Enhanced synchronized ultrasonic and flowfield fractionation of suspensions Ultrasonics 32 113-21

[23] Harris N R, Hill M, Beeby S, Shen Y, White N M, Hawkes J J and Coakley W T 2003 A silicon microfluidic ultrasonic separator Sensors Actuators B 95 425-34

[24] Yasuda K, Umemura S I and Takeda K 1996 Particle separation using acoustic radiation force and electrostatic force J. Acoust. Soc. Am. 99 1965-70
[25] Petersson F, Nilsson A, Holm C, Jonsson H and Laurell T 2005 Continuous separation of lipid particles from erythrocytes by means of laminar flow and acoustic standing wave forces Lab Chip 5 20-2

[26] Nilsson A, Petersson F, Jonsson H and Laurell T 2004 Acoustic control of suspended particles in micro fluidic chips Lab Chip 4 131-5

[27] Petersson F, Nilsson A, Holm C, Henrik J and Laurell T 2004 Separation of lipids from blood utilizing ultrasonic standing waves in microfluidic channels Analyst $129938-43$

[28] Yamada M, Nakashima M and Seki M 2004 Pinched flow fractionation: continuous size separation of particles utilizing a laminar flow profile in a pinched microchannel Anal. Chem. 76 5465-71

[29] Takagi J, Yamada M, Yasuda M and Seki M 2005 Continuous particle separation in a microchannel having asymmetrically arranged multiple branches Lab Chip 5 778-84

[30] Yamada M and Seki M 2005 Hydrodynamic filtration for on-chip particle concentration and classification utilizing microfluidics Lab Chip 5 1233-9

[31] Stemme E and Stemme G 1993 Valveless diffuser/nozzle-based fluid pump Sensors Actuators A 39 159-67

[32] Olsson A, Stemme G and Stemme E 1996 Diffuser-element design investigation for valve-less pumps Sensors Actuators A 57 137-43

[33] Olsson A, Enoksson P, Stemme G and Stemme E 1997 Micromachined flat-walled valveless diffuser pumps J. Microelectromech. Syst. 6 161-6

[34] Lee C J, Tsu Z K, Lei U, Hsu C J and Sheen H J 2005 A Valveless Micropump with Asymmetric Obstacles 16th Int. Sym. on Transport Phenomena (Prague, Czech, 29 Aug.-1 Sept.)

[35] Olsson A, Larsson O, Holm J, Lundbladh L, Ohman O and Stemme G 1998 Valve-less diffuser micropumps fabricated using thermoplastic replication Sensors Actuators A 64 63-8

[36] Uchida S 1950 The pulsating viscous flow superposed on the steady laminar motion of incompressible fluid in a circular pipe Z. Angew. Math. Phys. 720

[37] Hsu C J, Sheen H J, Wu T H, Chu H C, Chang C C and Lei U 2006 Characteristics of flow field in a PZT self-pumping micromixer Asia-Pacific Conf. of Transducers and Micro-Nano Technology (Singapore, 26-28 June)

[38] Yamanaka G, Kikura H, Takeda Y and Aritomi M 2004 Flow measurement on oscillating pipe flow near the entrance using the UVP method Exp. Fluids 32 212-20

[39] Meinhart C D, Wereley S T and Santiago J G 1999 PIV measurements of a microchannel flow Exp. Fluids 27 414-9

[40] Yang J T, Chen C K, Tsai K J, Lin W Z and Sheen H J 2006 A novel fluidic oscillator incorporating step-shaped attachment walls Sensors Actuators A at press

[41] Gravesen P, Branebjerg J and Jensen O S 1993 Microfluidic-a review J. Micromech. Microeng. 3 168-82 